\title{
Impact of Parthenium hysterophorus L. Invasion on Species Diversity of Cultivated Fields of Bilaspur (C.G.) India
}

\author{
Preeti Kumari, Pankaj K. Sahu, Madhu Y. Soni, Poonam Awasthi \\ Department of Botany, Dr. C.V. Raman University, Bilaspur (C.G.), India \\ Email: sahu.pankaj1@gmail.com
}

Received 31 May 2014; revised 30 June 2014; accepted 24 July 2014

Copyright ( 2014 by authors and Scientific Research Publishing Inc.

This work is licensed under the Creative Commons Attribution International License (CC BY). http://creativecommons.org/licenses/by/4.0/

(c) (i) Open Access

\begin{abstract}
In present study invasion of Parthenium hysterophorus $\mathrm{L}$. and its impacts on agriculture fields will be done in different cultivated crops in Bilaspur. The present work deals with diversity and distribution of weeds species in cultivated fields of Bilaspur C.G. A total number of 28 species under 27 genera and 16 families was distributed in three study sites (Koni, Sakri \& Kota) of cultivated fields in Bilaspur C.G. These study sites were located in different altitudinal zones responding different conditions in the this study. The number of species per sites ranged from 13 to 24 . In the Sakri, number of species was ranged from 17 to 27 , and the relative density of plant species ranged between 0.77 (Heteropogon contortus, Solanum surratense, Vernonia cinneria and Leucas aspera) and 22.92 (Parthenium hysterophorus). In the Kota, number of species was ranged from 14 to 28, and the relative density of plant species ranged between 0.85 (Ageratum conyzoides, Heteropogon contortus, Triticum aestivum) and 29.06 (Zea mays) and 30.91 (Parthenium hysterophorus). In the Agriculture field, number of species was ranged from 15 to 28, and the relative density of plant species ranged between 0.58 (Leucas aspera) and 26.23 (Triticum aestivum). Present work will give the information that weeds are harmful to herbs, crops and medicinal plants. Parthenium weed can suppress and crowd out other weed species, and could form a single dominant population in the invasion area, causing serious threat to plant community biological diversity.
\end{abstract}

\section{Keywords}

Parthenium, Invasion, Species Diversity, Crops, Bilaspur

\section{Introduction}

Invasive alien species are a serious implement to conservation and use of global biodiversity [1]. Parthenium

How to cite this paper: Kumari, P., Sahu, P.K., Soni, M.Y. and Awasthi, P. (2014) Impact of Parthenium hysterophorus L. Invasion on Species Diversity of Cultivated Fields of Bilaspur (C.G.) India. Agricultural Sciences, 5, 754-764.

http://dx.doi.org/10.4236/as.2014.58079 
hysterophorus, Eichornia crassipes and Lantana camara are among major IAS yet identified in Ethiopia [2]. Parthenium hysterophorus L. (Congress Grass) (here after referred as Parthenium) belongs to the family Asteraceae. Parthenium was introduced into Asia, Africa and Oceania with cereal and grass seed shipment from America during the 1950s [3]. Parthenium spreads easily through trade as contaminants of grain and other crop products by means of farm machineries [4]. In tropical and subtropical areas of the Indian, African and Australian continent, there is increasing concern on these adverse effects on human health, livestock, agriculture productivity and biodiversity [5]. Manual cutting results in rapid regeneration, which is quickly followed by flowering with abundant seed production [6].

The weed looks similar to other introduced weeds from the same family including seedlings of cobbler's pegs, leaves of Artemisia spp., flowers of bishop's weed Ammi majus and Conium maculatum and Ambrosia sp. [7]. In present study, work was done on impact of Parthenium, on species diversity, composition, agriculture in different cultivated crops of Bilaspur, C.G.

\section{Materials \& Methods}

\subsection{Study Area}

The study was carried out in three study sites (Koni, Sakri \& Kota) of cultivated fields of Bilaspur Chhattisgarh. The area experiences a typical monsoon climate, with three distinct seasons: summer from March-June, rainy monsoon period from July-October and winter from November-February. Bilaspur is situated between $17^{\circ}$ to $23.7^{\circ}$ north latitude and $8.40^{\circ}$ to $83.38^{\circ}$ east longitude. The climate is mainly tropical, humid and sub-humid. The average annual rainfall of the region is around $1400 \mathrm{~mm}$ and about $90 \%$ to $95 \%$ of this amount is received during south-west monsoon season (June-October). The atmospheric humidity is very high $(>90 \%)$ during monsoon months and starts decreasing from October onwards and reaches as low as $15 \%-20 \%$ during peak summer months. The soils of Chhattisgarh vary considerably in the three agro-climatic zones.

\subsection{Topography and Soil}

The landscape of the Bilaspur represents a complex of geological structure. This zone has a landscape whose physical property ranges from flat to gentle slope and hilly. Study areas are highly populated, rapidly urbanizing as industrial and market development centers. Productive agricultural land is rapidly being converted into housing areas in both study sites. The soils are generally lateritic, nutrient-poor and characterized by excessive amounts of iron oxide. In the present study area alluvial soils are found along the streams and rivers. The rock types are schist and gneisses with granite intrusion, sandstones, shales, limestone, basaltic lava and laterite with bauxite.

\subsection{Data Collection}

The study was carried out in 3 different sites of Bilaspur Chhattisgarh. The area experiences a typical monsoon climate, with three distinct seasons: summer from March-June, rainy monsoon period from July-October and winter from November-February. The soils are generally lateritic, nutrient-poor and characterized by excessive amounts of iron oxide. In the present study area alluvial soils are found along the streams and rivers. Field experiments were conducted to assess the impact of Parthenium weed (Parthenium hysterophorus L.) on species diversity of herbs and ground vegetation in cropped area of, Kota, Koni and Sakri sites in Bilaspur District, during 2013-2014. The samples were taken at the interval of $3 \mathrm{~km}$. A total of 90 quadrats in each 3 sample sites (Kota, Sakri and Koni), thirty sample quadrats were taken from each major site. Each measuring $1 \mathrm{~m} \times 1 \mathrm{~m}\left(1 \mathrm{~m}^{2}\right)$ sample quadrate was laid to assess the impact of Parthenium on aboveground herbaceous vegetation cover in the study area. Weeds species and herbaceous species were identified with help of Herbarium and local flora of state. The data on species diversity were analyzed for density and frequency [8]. The importance value index (IVI) was determined as the sum of relative frequency, and relative density [9]. Relative frequency and relative density relative were determined following Phillips [10].

\section{Observation \& Results}

The total of 25, 26 and 28 species was recorded in Koni, Sakri \& Kota sites, respectively. There were less spe- 
cies in the invaded areas (23, respectively) than in the non-invaded areas (29, respectively) in 3 sites. Most common species found were Achyranthes aspera, Ageratum conyzoides, Allium cepa, Argemone mexicana, Cajanus cajans, Cynadon dactylon, Dolichous, Tridax procumbans, Triticum aestivum, Xanthium, Zea mays etc. in all studied areas. In all 3 sites some extra species were found in non-invaded areas like Alternanthera, Blumea, Borreria, Commellina, and Vernonia etc. Significant decreased in species richness found from Parthenium noninvaded to invaded areas. The species richness decreased on increase of Parthenium density in all study areas.

A total number of 28 species under 27 genera and 16 families distributed in three major study sites of cultivated fields of Bilaspur C.G. These study sites were located in different altitudinal zones responding digfferent condition in study sites, the number of species per sites ranged from 13 (KT4) to 24 (SK1). In the Agriculture field, number of species were ranged from 15 to 28, and the relative density of plant species ranged between 0.58 (Leucas aspera) and 26.23 (Triticum aestivum). The relative density of most of weeds in cultivated fields were 2.86 (18 species) and RD lower than 2 were observed in (11 species). In accordance with the variation in frequency/relative frequency \& density relative density, the IVI values varied from 2.91 to 35.60 . There were 9 species with IVI lower than 4 (Table 1, Table 2).

In the Sakri, number of species were ranged from 17 to 27, and the relative density of plant species ranged between 0.77 (Heteropogon contortus, Solanum surratense, Vernonia cinneria and Leucas aspera) aspera and 22.92 (Parthenium hysterophorus). The relative density of weeds of cultivated fields were 0.81 (7 species) and there were RD lower than 1 (8 species). In accordance with the variation in frequency/relative frequency \& density or relative density, the IVI values varied from 2.07 to 35.42 . There were 12 species with IVI lower than 10 (Table 3, Table 4).

In the Kota, number of species were ranged from 14 to 28, and the relative density of plant species ranged between 0.85 (Ageratum conyzoides, Heteropogon contortus, Triticum aestivum) and 29.06 (Zea mays) and 30.91 (Parthenium hysterophorus). The relative density of weeds of cultivated fields were $0.81-0.97$ (5 species) and there were RD lower than 2 (10 species). In accordance with the variation in frequency/relative frequency \& density relative density, the IVI values varied from 3.98 to 35.00 . There were 11 species with IVI lower than 5 (Table 5, Table 6).

There were 3 major sites studied Koni, Sakari \& Kota at Bilaspur. These sites shows the diverse and distribution pattern in frequency, density weeds species in Parthenium invaded and non invaded sites of Bilaspur. The gradual increase of Parthenium coverage towards the core of Agriculture areas and perennial barren and wastelands was found from the spatial observation and frequency distribution of site survey, in all study areas. Similarly, the gradual increase of Parthenium cover was found up to frequency class (50\% - 100\%). The invasion found increasing towards the semi-urbanized areas, agricultural lands and margin of forest areas.

\section{Discussion}

The dominant weeds were found in invaded sites of Parthenium (Achyranthes aspera, Argemone mexicana, Tridax procumbans Cynodon dactylon, Dichanthium annulatum Allium cepa, Zea mays, Dichanthium annulatum) while in non-invaded sites dominant species were Triticum aestivum, Tridax procumbans Dolichos lablab, Achyranthes aspera Ageratum conyzoides Allium cepa and Dichanthium annulatum while less dominant were Xanthium strumarium, Borreria stricta and Blumea sp. in study sites of cultivated crops of Bilaspur C.G. The similarity indices between Parthenium non-invaded and invaded areas of both study areas were found high. The high similarity value between Parthenium non-invaded and invaded areas of all study sites indicated that there is no radical change in species composition within the area.

Kohli et al. [11] reported a decline in species richness from 25 to 12 from Parthenium non-invaded site to high invaded site of Lower Himalaya (India). In present work the species diversity were observed from 28 (Non invaded site) to 13 (Invaded site). Similarly, Adkins and Sowerby [12] experimentally proved that Parthenium has allelopathic effect on its root and shoot leachates and thus has the ability to reduce the growth and germination of numerous associated species. The Xanthium, Achyranthes, Tridex might have strong association or competitive vigour with Parthenium; hence it was present only in Parthenium invaded area. But, Ageratum, Borreria, Allium cepa, Cajans, Digitaria ciliaris, Cynadon, Dolichous, Triticum, Zea mays were negatively affected by Parthenium invasion. The dominant weeds were found in invaded sites of Parthenium (Zea mays, Cynodon dactylon) while co-dominant species were Digitalia ciliaris, Dolichos lablab and in non-invaded sites dominant species was Triticum aestivum while less dominant one was Leucas aspera in agriculture field of Bilaspur C.G. 
Table 1. Representing the RD of invaded and non-invaded sites of Parthenium hysterophorus in Agriculture field (Koni).

\begin{tabular}{|c|c|c|c|c|c|c|c|c|c|c|}
\hline & \multicolumn{5}{|c|}{ Parthenium Non-invaded } & \multicolumn{5}{|c|}{ Parthenium Invaded } \\
\hline & KN1 & KN2 & KN3 & KN4 & KN5 & KN1 & KN2 & KN3 & KN4 & KN5 \\
\hline Achyranthes aspera & 3.49 & 3.66 & 3.28 & 3.94 & 8.57 & & 2.00 & 4.46 & 4.17 & 7.41 \\
\hline Anagallis arvensis & 8.72 & 3.05 & 5.74 & & 2.86 & & & & & \\
\hline Ageratum conyzoides & 2.91 & 3.05 & 5.74 & 3.15 & 2.86 & 6.67 & 4.00 & 2.68 & 4.17 & 3.70 \\
\hline Allium сера & & & & 3.94 & 2.86 & 6.67 & 11.00 & 16.96 & 12.50 & 7.41 \\
\hline Alternanthera paronychioides & 4.65 & 1.83 & & & 2.86 & & & & & \\
\hline Argemone mexicana & 8.72 & 4.27 & 6.56 & 4.72 & 2.86 & 3.33 & 6.00 & 2.68 & 1.39 & 3.70 \\
\hline Blumea lacera & & 1.22 & & & 2.86 & & & & & \\
\hline Borreria stricta & 0.58 & & & & 2.86 & & & & & \\
\hline Cajanus cajan & 5.81 & & 3.28 & 6.30 & 5.71 & 6.67 & 11.00 & 8.93 & 6.94 & 7.41 \\
\hline Chrysophogon aciculatus & 4.65 & 5.49 & 4.10 & & 2.86 & 6.67 & 4.00 & 3.57 & 2.78 & 3.70 \\
\hline Commelina benghalensis & & & & & 2.86 & & & & & \\
\hline Cynodon dactylon & 9.88 & 6.71 & 3.28 & 5.51 & 5.71 & 6.67 & 3.00 & 7.14 & 12.50 & 3.70 \\
\hline Desmodium triflorum & 1.74 & 4.88 & & 4.72 & 2.86 & 6.67 & 4.00 & 3.57 & 1.39 & 3.70 \\
\hline Dichanthium annulatum & 5.81 & 6.71 & 11.48 & 3.94 & 2.86 & 3.33 & 2.00 & 3.57 & 5.56 & 7.41 \\
\hline Dichanthium aristatum & & & & & & & 3.00 & 2.68 & 2.78 & 3.70 \\
\hline Digitaria ciliaris & 2.91 & 3.05 & 5.74 & & 5.71 & 3.33 & 4.00 & 6.25 & 4.17 & 7.41 \\
\hline Dolichos lablab & 6.40 & 7.32 & 2.46 & 3.94 & 2.86 & 6.67 & 11.00 & 3.57 & 2.78 & 3.70 \\
\hline Heteropogon contortus & 7.56 & 9.15 & & 5.51 & 2.86 & & & & & \\
\hline Leucas aspera & 0.58 & & & & 2.86 & & & & & \\
\hline Lindernia crustata & 1.16 & 6.71 & 9.02 & 3.94 & 5.71 & & & & & \\
\hline Parthenium hysterophorus & & & & & & 6.67 & 9.00 & 16.96 & 18.06 & 11.11 \\
\hline Raphanus sativus & 6.40 & 1.83 & 4.92 & 7.09 & 2.86 & 6.67 & 4.00 & 2.68 & 4.17 & 3.70 \\
\hline Solanum surratense & 2.91 & 6.10 & 3.28 & 4.72 & 2.86 & 3.33 & 3.00 & 0.89 & 1.39 & 3.70 \\
\hline Tridax procumbans & 1.16 & 3.66 & 4.92 & 2.36 & 5.71 & 10.00 & & & & \\
\hline Triticum aestivum & 10.47 & 12.20 & 26.23 & 21.26 & 5.71 & 6.67 & & & & \\
\hline Vernonia cinneria & & 1.83 & & & 2.86 & & & & & \\
\hline Xanthium strumarium & 2.33 & 5.49 & & 3.15 & 5.71 & 3.33 & 9.00 & 4.46 & 4.17 & 7.41 \\
\hline Zea mays & 1.16 & 1.83 & & 11.81 & 2.86 & 6.67 & 10.00 & 8.93 & 11.11 & 11.11 \\
\hline
\end{tabular}


Table 2. Representing the IVI of invaded and non-invaded of Parthenium hysterophorus in study sites of agriculture fields (Koni).

\begin{tabular}{|c|c|c|c|c|c|c|c|c|c|c|}
\hline \multirow{2}{*}{ Name of plant species } & \multicolumn{5}{|c|}{ Parthenium Non-invaded Sites } & \multicolumn{5}{|c|}{ Parthenium Invaded Sites } \\
\hline & KN1 & KN2 & KN3 & KN4 & KN5 & KN1 & KN2 & KN3 & KN4 & KN5 \\
\hline Achyranthes aspera & 10.47 & 6.04 & 9.53 & 6.97 & 12.12 & & 7.71 & 7.32 & 8.01 & 11.41 \\
\hline Anagallis arvensis & 15.70 & 7.81 & 8.86 & & 4.28 & & & & & \\
\hline Ageratum conyzoides & 7.56 & 5.43 & 11.99 & 9.21 & 4.28 & 10.04 & 6.86 & 8.39 & 11.86 & 6.37 \\
\hline Allium сера & & & & 10.00 & 4.28 & 15.66 & 16.71 & 25.54 & 20.19 & 22.07 \\
\hline Alternanthera paronychioides & 9.30 & 4.21 & & & 4.98 & & & & & \\
\hline Argemone mexicana & 15.70 & 11.41 & 12.81 & 10.79 & 4.28 & 5.58 & 11.71 & 5.54 & 5.24 & 7.70 \\
\hline Blumea lacera & & 3.60 & & & 5.69 & & & & & \\
\hline Borreria stricta & 2.91 & & & & 3.57 & & & & & \\
\hline Cajanus cajan & 10.47 & & 9.53 & 12.36 & 11.39 & 19.03 & 16.71 & 14.64 & 14.64 & 18.07 \\
\hline Chrysophogon aciculatus & 9.30 & 12.63 & 7.22 & & 3.57 & 10.04 & 9.71 & 9.29 & 6.62 & 9.04 \\
\hline Commelina benghalensis & & & & & 4.28 & & & & & \\
\hline Cynodon dactylon & 16.86 & 11.47 & 9.53 & 8.54 & 9.97 & 10.04 & 8.71 & 12.86 & 24.04 & 6.37 \\
\hline Desmodium triflorum & 4.07 & 9.64 & & 10.79 & 3.57 & 8.91 & 9.71 & 9.29 & 5.24 & 5.04 \\
\hline Dichanthium annulatum & 10.47 & 11.47 & 20.85 & 10.00 & 4.98 & 4.46 & 7.71 & 9.29 & 9.40 & 10.07 \\
\hline Dichanthium aristatum & & & & & & & 8.71 & 8.39 & 10.47 & 6.37 \\
\hline Digitaria ciliaris & 7.56 & 7.81 & 15.11 & & 9.26 & 4.46 & 9.71 & 14.82 & 8.01 & 16.74 \\
\hline Dolichos lablab & 11.05 & 12.08 & 8.71 & 10.00 & 4.28 & 23.52 & 16.71 & 9.29 & 6.62 & 6.37 \\
\hline Heteropogon contortus & 12.21 & 16.29 & & 11.57 & 6.40 & & & & & \\
\hline Leucas aspera & 2.91 & & & & 3.57 & & & & & \\
\hline Lindernia crustata & 5.81 & 13.85 & 18.39 & 10.00 & 7.84 & & & & & \\
\hline Parthenium hysterophorus & & & & & & 25.77 & 14.71 & 25.54 & 29.59 & 25.78 \\
\hline Raphanus sativus & 11.05 & 4.21 & 14.29 & 13.15 & 4.98 & 12.28 & 9.71 & 8.39 & 8.01 & 7.70 \\
\hline Solanum surratense & 5.23 & 10.86 & 6.40 & 10.79 & 4.28 & 6.70 & 8.71 & 3.75 & 5.24 & 5.04 \\
\hline Tridax procumbans & 3.49 & 8.42 & 11.17 & 5.39 & 9.26 & 14.49 & & & & \\
\hline Triticum aestivum & 17.44 & 19.34 & 35.60 & 30.35 & 14.93 & 10.04 & & & & \\
\hline Vernonia cinneria & & 6.59 & & & 3.57 & & & & & \\
\hline Xanthium strumarium & 4.65 & 10.25 & & 9.21 & 8.55 & 5.58 & 17.57 & 10.18 & 8.01 & 12.74 \\
\hline Zea mays & 5.81 & 6.59 & & 20.90 & 3.57 & 13.41 & 18.57 & 17.50 & 18.80 & 23.11 \\
\hline
\end{tabular}


Table 3. Representing the RD of invaded and non-invaded sites of Parthenium hysterophorus in Sakri.

\begin{tabular}{|c|c|c|c|c|c|c|c|c|c|c|}
\hline \multirow{2}{*}{ Name of species } & \multicolumn{5}{|c|}{ Parthenium Non-invaded Sites } & \multicolumn{5}{|c|}{ Parthenium Invaded Sites } \\
\hline & SK1 & SK2 & SK3 & SK4 & SK5 & SK1 & SK2 & SK3 & SK4 & SK5 \\
\hline Achyranthes aspera & 1.61 & 3.48 & 5.38 & 5.13 & 8.64 & 6.15 & 3.85 & 3.70 & 4.17 & 3.57 \\
\hline Anagallis arvensis & 1.61 & 0.87 & 1.54 & 2.56 & 2.47 & & & & & \\
\hline Ageratum conyzoides & 0.81 & 3.48 & 2.31 & 5.13 & 3.70 & 6.15 & 3.85 & 1.85 & 4.17 & 2.38 \\
\hline Allium сера & 2.42 & 6.09 & 9.23 & & 6.17 & 15.38 & 15.38 & 12.96 & 10.42 & 8.33 \\
\hline Alternanthera paronychioides & & 2.61 & & 8.55 & 3.70 & & & & & \\
\hline Argemone mexicana & 5.65 & 0.87 & 3.08 & 0.85 & 4.94 & 3.08 & 1.92 & 1.85 & 2.08 & 1.19 \\
\hline Blumea lacera & 1.61 & & 1.54 & 2.56 & & & & & & \\
\hline Borreria stricta & 0.81 & 2.61 & 3.08 & 4.27 & 4.94 & & & & & \\
\hline Cajanus cajan & 8.87 & 1.74 & 3.08 & & 3.70 & 3.08 & 3.85 & 1.85 & 4.17 & 7.14 \\
\hline Chrysophogon aciculatus & 0.81 & 9.57 & 3.08 & 5.98 & 2.47 & 4.62 & 1.92 & 1.85 & 2.08 & 1.19 \\
\hline Commelina benghalensis & & 2.61 & 3.85 & 1.71 & & & & & & \\
\hline Cynodon dactylon & 8.87 & 11.30 & 12.31 & 8.55 & 6.17 & 13.85 & 7.69 & 16.67 & 8.33 & 5.95 \\
\hline Desmodium triflorum & 1.61 & 1.74 & & 1.71 & 4.94 & 7.69 & 1.92 & 3.70 & 4.17 & 1.19 \\
\hline Dichanthium annulatum & 0.81 & 2.61 & 3.08 & 0.85 & 4.94 & 7.69 & 5.77 & 1.85 & 4.17 & 4.76 \\
\hline Dichanthium aristatum & & & & & & 1.54 & 3.85 & 3.70 & 2.08 & 3.57 \\
\hline Digitaria ciliaris & 4.84 & 0.87 & 1.54 & 1.71 & 2.47 & 3.08 & 3.85 & 3.70 & 4.17 & 2.38 \\
\hline Dolichos lablab & 10.48 & 9.57 & 16.15 & 13.68 & 12.35 & 4.62 & 1.92 & 3.70 & 8.33 & 15.48 \\
\hline Heteropogon contortus & 0.81 & 2.61 & 0.77 & 0.85 & 2.47 & & & & & \\
\hline Leucas aspera & 0.81 & 0.87 & 0.77 & 3.42 & & & & & & \\
\hline Lindernia crustata & 1.61 & & & 2.56 & 3.70 & & & & & \\
\hline Parthenium hysterophorus & & & & & & 10.77 & 17.31 & 22.22 & 22.92 & 15.48 \\
\hline Raphanus sativus & 6.45 & 6.96 & 1.54 & 1.71 & 3.70 & 4.62 & 1.92 & 3.70 & 2.08 & 4.76 \\
\hline Solanum surratense & 3.23 & 1.74 & 0.77 & 4.27 & 4.94 & 1.54 & 5.77 & 1.85 & 4.17 & 2.38 \\
\hline Tridax procumbans & 14.52 & 6.96 & 7.69 & 2.56 & 6.17 & & & & & \\
\hline Triticum aestivum & 10.48 & 17.39 & 9.23 & 6.84 & 2.47 & & & & & \\
\hline Vernonia cinneria & 2.42 & 1.74 & 0.77 & 1.71 & 1.23 & & & & & \\
\hline Xanthium strumarium & 0.81 & 0.00 & 3.85 & 1.71 & 0.00 & 3.08 & 5.77 & 3.70 & 4.17 & 5.95 \\
\hline Zea mays & 8.06 & 1.74 & 5.38 & 11.11 & 3.70 & 3.08 & 13.46 & 11.11 & 8.33 & 14.29 \\
\hline
\end{tabular}


Table 4. Representing the IVI of invaded and non-invaded of Parthenium hysterophorus in study sites of Sakri.

\begin{tabular}{|c|c|c|c|c|c|c|c|c|c|c|}
\hline \multirow{2}{*}{ Name of plant species } & \multicolumn{5}{|c|}{ Parthenium Non-invaded Sites } & \multicolumn{5}{|c|}{ Parthenium Invaded Sites } \\
\hline & SK1 & SK2 & SK3 & SK4 & SK5 & SK1 & SK2 & SK3 & SK4 & SK5 \\
\hline Achyranthes aspera & 4.18 & 8.61 & 7.98 & 12.82 & 14.70 & 10.00 & 7.85 & 7.41 & 8.33 & 7.14 \\
\hline Anagallis arvensis & 4.18 & 3.43 & 2.84 & 5.13 & 5.50 & & & & & \\
\hline Ageratum conyzoides & 3.37 & 11.17 & 3.61 & 10.26 & 6.73 & 10.00 & 7.85 & 5.56 & 12.50 & 5.95 \\
\hline Allium сера & 7.55 & 11.22 & 13.13 & 0.00 & 12.23 & 26.92 & 27.38 & 24.07 & 22.92 & 15.48 \\
\hline Alternanthera paronychioides & 0.00 & 5.17 & 0.00 & 16.24 & 6.73 & & & & & \\
\hline Argemone mexicana & 10.77 & 3.43 & 4.38 & 3.42 & 11.00 & 6.92 & 5.92 & 5.56 & 6.25 & 4.76 \\
\hline Blumea lacera & 4.18 & 0.00 & 2.84 & 5.13 & & & & & & \\
\hline Borreria stricta & 3.37 & 5.17 & 5.67 & 9.40 & 11.00 & & & & & \\
\hline Cajanus cajan & 16.56 & 4.30 & 5.67 & & 6.73 & 6.92 & 7.85 & 5.56 & 8.33 & 14.29 \\
\hline Chrysophogon aciculatus & 3.37 & 17.26 & 5.67 & 13.68 & 8.53 & 12.31 & 5.92 & 5.56 & 6.25 & 4.76 \\
\hline Commelina benghalensis & & 5.17 & 5.14 & 6.84 & 0.00 & & & & & \\
\hline Cynodon dactylon & 14.00 & 19.00 & 16.20 & 16.24 & 12.23 & 25.38 & 15.69 & 24.07 & 16.67 & 13.10 \\
\hline Desmodium triflorum & 4.18 & 4.30 & 0.00 & 4.27 & 7.97 & 15.38 & 5.92 & 7.41 & 8.33 & 4.76 \\
\hline Dichanthium annulatum & 3.37 & 7.74 & 4.38 & 3.42 & 11.00 & 15.38 & 13.77 & 5.56 & 8.33 & 11.90 \\
\hline Dichanthium aristatum & & & & & & 5.38 & 7.85 & 7.41 & 6.25 & 7.14 \\
\hline Digitaria ciliaris & 9.97 & 3.43 & 2.84 & 4.27 & 5.50 & 6.92 & 7.85 & 11.11 & 8.33 & 5.95 \\
\hline Dolichous lablab & 18.18 & 17.26 & 43.43 & 18.80 & 21.44 & 8.46 & 5.92 & 11.11 & 12.50 & 26.19 \\
\hline Heteropogon contortus & 3.37 & 5.17 & 2.07 & 3.42 & 5.50 & & & & & \\
\hline Leucas aspera & 3.37 & 3.43 & 2.07 & 5.98 & 0.00 & & & & & \\
\hline Lindernia crustata & 4.18 & 0.00 & 0.00 & 5.13 & 9.76 & & & & & \\
\hline Parthenium hysterophorus & & & & & & 18.46 & 25.31 & 33.33 & 35.42 & 26.19 \\
\hline Raphanus sativus & 11.58 & 12.08 & 2.84 & 4.27 & 6.73 & 12.31 & 5.92 & 7.41 & 6.25 & 8.33 \\
\hline Solanum surratense & 5.79 & 4.30 & 2.07 & 9.40 & 7.97 & 5.38 & 9.77 & 5.56 & 8.33 & 5.95 \\
\hline Tridax procumbans & 22.21 & 14.65 & 20.68 & 5.13 & 9.20 & & & & & \\
\hline Triticum aestivum & 18.18 & 25.08 & 19.62 & 11.97 & 5.50 & & & & & \\
\hline Vernonia cinneria & 4.98 & 4.30 & 2.07 & 4.27 & 4.26 & & & & & \\
\hline Xanthium strumarium & 3.37 & 0.00 & 10.34 & 4.27 & 0.00 & 6.92 & 13.77 & 11.11 & 8.33 & 13.10 \\
\hline Zea mays & 15.76 & 4.30 & 14.48 & 16.24 & 9.76 & 6.92 & 25.46 & 22.22 & 16.67 & 25.00 \\
\hline
\end{tabular}


Table 5. Representing the RD of invaded and non-invaded sites of Parthenium hysterophorus in Kota.

\begin{tabular}{|c|c|c|c|c|c|c|c|c|c|c|}
\hline \multirow{2}{*}{ Name of species } & \multicolumn{5}{|c|}{ Parthenium Non-invaded Sites } & \multicolumn{5}{|c|}{ Parthenium Invaded Sites } \\
\hline & KT1 & KT2 & KT3 & KT4 & KT5 & KT1 & KT2 & KT3 & KT4 & KT5 \\
\hline Achyranthes aspera & 6.10 & 5.75 & 5.83 & 9.40 & 6.14 & 4.44 & 10.71 & 3.45 & 10.87 & 3.64 \\
\hline Anagallis arvensis & 3.66 & 1.15 & 1.94 & 3.42 & 4.39 & & & & & \\
\hline Ageratum conyzoides & 7.32 & 5.75 & 8.74 & 0.85 & 4.39 & 8.89 & 3.57 & 6.90 & 0.00 & 3.64 \\
\hline Allium сера & 9.76 & 1.15 & 9.71 & 2.56 & 13.16 & 0.00 & 3.57 & 3.45 & 10.87 & 5.45 \\
\hline Alternanthera paronychioides & 4.88 & 3.45 & 5.83 & 3.42 & 1.75 & & & & & \\
\hline Argemone mexicana & 2.44 & 4.60 & 0.97 & 1.71 & 1.75 & 4.44 & 0.00 & 3.45 & 6.52 & 5.45 \\
\hline Blumea lacera & 1.22 & 2.30 & 7.77 & 1.71 & 2.63 & & & & & \\
\hline Borreria stricta & 6.10 & 0.00 & 0.00 & 3.42 & 4.39 & & & & & \\
\hline Cajanus cajan & 4.88 & 10.34 & 2.91 & 0.00 & 0.88 & 4.44 & 1.79 & 1.72 & 2.17 & 1.82 \\
\hline Chrysophogon aciculatus & 1.22 & 1.15 & 0.00 & 5.13 & 2.63 & 0.00 & 5.36 & 5.17 & 13.04 & 1.82 \\
\hline Commelina benghalensis & 4.88 & 0.00 & 4.85 & 0.00 & 1.75 & & & & & \\
\hline Cynodon dactylon & 4.88 & 4.60 & 1.94 & 3.42 & 1.75 & 4.44 & 1.79 & 1.72 & 8.70 & 9.09 \\
\hline Desmodium triflorum & 8.54 & 10.34 & 0.97 & 2.56 & 2.63 & 0.00 & 3.57 & 6.90 & 4.35 & 3.64 \\
\hline Dichanthium annulatum & 2.44 & 5.75 & 4.85 & 1.71 & 2.63 & 2.22 & 10.71 & 1.72 & 10.87 & 1.82 \\
\hline Dichanthium aristatum & & & & & 3.51 & 11.11 & 1.79 & 1.72 & 0.00 & 7.27 \\
\hline Digitaria ciliaris & 2.44 & 6.90 & 0.00 & 4.27 & 3.51 & 11.11 & 0.00 & 6.90 & 8.70 & 1.82 \\
\hline Dolichos lablab & 3.66 & 2.30 & 1.94 & 2.56 & 4.39 & 2.22 & 5.36 & 3.45 & 0.00 & 0.00 \\
\hline Heteropogon contortus & 1.22 & 6.90 & 0.97 & 0.85 & 3.51 & & & & & \\
\hline Leucas aspera & 2.44 & 0.00 & 3.88 & 2.56 & 3.51 & & & & & \\
\hline Lindernia crustata & 2.44 & 4.60 & 2.91 & 2.56 & 6.14 & & & & & \\
\hline Parthenium hysterophorus & & & & & 2.63 & 8.89 & 10.71 & 10.34 & 6.52 & 30.91 \\
\hline Raphanus sativus & 1.22 & 2.30 & 0.00 & 1.71 & 5.26 & 2.22 & 0.00 & 0.00 & 4.35 & 5.45 \\
\hline Solanum surratense & 1.22 & 3.45 & 9.71 & 4.27 & 3.51 & 4.44 & 16.07 & 13.79 & 0.00 & 1.82 \\
\hline Tridax procumbans & 4.88 & 3.45 & 0.00 & 5.98 & 2.63 & 4.44 & 7.14 & 1.72 & 4.35 & 1.82 \\
\hline Triticum aestivum & 2.44 & 0.00 & 6.80 & 0.85 & 1.75 & 6.67 & 0.00 & 12.07 & 4.35 & 3.64 \\
\hline Vernonia cinneria & 2.44 & 5.75 & 4.85 & 1.71 & 8.77 & & & & & \\
\hline Xanthium strumarium & 2.44 & 0.00 & 3.88 & 4.27 & 10.0 & 0.00 & 5.36 & 8.62 & 0.00 & 5.45 \\
\hline Zea mays & 4.88 & 8.05 & 8.74 & 29.06 & & 20.00 & 12.50 & 6.90 & 4.35 & 5.45 \\
\hline
\end{tabular}


Table 6. Representing the IVI of invaded and non-invaded of Parthenium hysterophorus in study sites of Kota.

\begin{tabular}{|c|c|c|c|c|c|c|c|c|c|c|}
\hline \multirow{2}{*}{ Name of plant species } & \multicolumn{5}{|c|}{ Parthenium Non-invaded Sites } & \multicolumn{5}{|c|}{ Parthenium Invaded Sites } \\
\hline & KT1 & KT2 & KT3 & KT4 & KT5 & KT1 & KT2 & KT3 & KT4 & KT5 \\
\hline Achyranthes aspera & 12.35 & 13.15 & 15.20 & 18.78 & 11.14 & 9.44 & 20.24 & 11.14 & 19.96 & 10.78 \\
\hline Anagallis arvensis & 6.78 & 4.85 & 5.07 & 6.54 & 9.39 & & & & & \\
\hline Ageratum conyzoides & 13.57 & 9.45 & 18.11 & 3.98 & 6.89 & 18.89 & 8.33 & 10.74 & & 7.21 \\
\hline Allium сера & 16.01 & 4.85 & 15.96 & 5.69 & 18.16 & 5.00 & 8.33 & 7.29 & 19.96 & 12.60 \\
\hline Alternanthera paronychioides & 8.00 & 7.15 & 8.95 & 6.54 & 4.25 & & & & & \\
\hline Argemone mexicana & 8.69 & 8.30 & 4.10 & 4.83 & 6.75 & 9.44 & & 11.14 & 11.07 & 12.60 \\
\hline Blumea lacera & 4.34 & 6.00 & 14.02 & 4.83 & 7.63 & & & & & \\
\hline Borreria stricta & 9.22 & & & 6.54 & 9.39 & & & & & \\
\hline Cajanus cajan & 8.00 & 17.75 & 9.16 & & 3.38 & 9.44 & 6.55 & 5.57 & 6.72 & 5.39 \\
\hline Chrysophogon aciculatus & 4.34 & 4.85 & & 11.38 & 7.63 & & 10.12 & 12.86 & 22.13 & 5.39 \\
\hline Commelina benghalensis & 8.00 & & 11.10 & & 4.25 & & & & & \\
\hline Cynodon dactylon & 8.00 & 8.30 & 5.07 & 9.67 & 4.25 & 9.44 & 6.55 & 5.57 & 13.24 & 16.23 \\
\hline Desmodium triflorum & 11.66 & 14.05 & 4.10 & 5.69 & 5.13 & & 8.33 & 14.59 & 8.89 & 10.78 \\
\hline Dichanthium annulatum & 5.56 & 9.45 & 11.10 & 4.83 & 7.63 & 7.22 & 20.24 & 5.57 & 19.96 & 5.39 \\
\hline Dichanthium aristatum & & & & & & 16.11 & 6.55 & 5.57 & & 14.42 \\
\hline Digitaria ciliaris & 5.56 & 10.60 & & 7.40 & 6.01 & 16.11 & & 14.59 & 17.79 & 5.39 \\
\hline Dolichous lablab & 6.78 & 6.00 & 5.07 & 5.69 & 8.51 & 7.22 & 10.12 & 7.29 & & \\
\hline Heteropogon contortus & 4.34 & 14.30 & 4.10 & 3.98 & 6.89 & & & & & \\
\hline Leucas aspera & 8.69 & & 7.01 & 5.69 & 6.01 & & & & & \\
\hline Lindernia crustata & 5.56 & 8.30 & 6.04 & 5.69 & 6.01 & & & & & \\
\hline Parthenium hysterophorus & & & & & & 13.89 & 20.24 & 18.04 & 11.07 & 41.62 \\
\hline Raphanus sativus & 4.34 & 6.00 & & 4.83 & 11.14 & 7.22 & & & 13.44 & 12.60 \\
\hline Solanum surratense & 4.34 & 7.15 & 15.96 & 7.40 & 5.13 & 9.44 & 25.60 & 21.49 & & 5.39 \\
\hline Tridax procumbans & 8.00 & 7.15 & & 9.11 & 10.26 & 9.44 & 11.90 & 5.57 & 8.89 & 5.39 \\
\hline Triticum aestivum & 5.56 & & 9.92 & 3.98 & 6.01 & 16.67 & 0.00 & 15.92 & 13.44 & 7.21 \\
\hline Vernonia cinneria & 5.56 & 13.15 & 7.98 & 4.83 & 7.63 & & & & & \\
\hline Xanthium strumarium & 5.56 & & 10.13 & 13.65 & 4.25 & & 14.88 & 12.47 & & 9.03 \\
\hline Zea mays & 11.13 & 19.16 & 11.86 & 38.43 & 16.27 & 35.00 & 22.02 & 14.59 & 13.44 & 12.60 \\
\hline
\end{tabular}


Displacement by direct competition reduced structural diversity, and increased biomass production and disruption of the prevailing vegetation dynamics [13]. Timsina [14] reported Trifolium repens, Imperata sp., Chrysopogan aciculatus, Sporobolus sp. and Dactyloctenium aegypticum as affected by Parthenium invasion and abundance of palatable species decreased with possible impact on fodder supply. The frequency of Triticum astevim increased from non invaded plots at Sakari and the frequency was nearly equal at invaded and non-invaded plots at Koni. Similarly, the nearly equal frequency of Allium cepa Dollichous, Cynadon at invaded and non-invaded plots indicates the resistance of Argemon, Desmodium against Parthenium invasion. There were no significant differences in frequency of Chrysopogan aciculatus, Cynodon dactylon, Mimosa pudica, Paspalidium flavidum, Paspalum scrobiculatum, Scoparia dulcis, Setaria gauca, Solanum surattense and Sida rhombifolia on Parthenium invasion, which meant that those species might compete with Parthenium. Timsina [14] reports similar abundance of Euphorbia hirta and Cassia tora in areas with different levels of Parthenium invasion. As reported by Mahadevappa, et al. [15] Parthenium weed caused up to 90\% decline in forage production. The survey was conducted in India. The species like Cassia sericea, Cassia tora, Cassia auriculata, Croton bonplandianum, Amaranthus spinosus, Tephrosia purpurea, Hyptis suaveolens, Sida spinosa and Mirabilis jalapa suppressed Parthenium in natural habitats shown by [16]. In Ethiopia it showed that about 93.6\%, 90.8\% and $77.7 \%$ of variation in density of broad leaved, grass and sedges were respectively accounted for the density of parthenium [17]. Partenium hysterophorus L., commonly known as congress grass, feverfew, ragweed parthenium or white top is a noxious weed native to tropical America. It has now naturalized in several tropical and subtropical parts of the world [18] [19]. The invasion is more favoured by the human activities than the allelopathic nature and reproductive efficiency of weeds invasion. Many aggressive invasive plants like Ageratum mexicana, Lantana camara and Parthenium hysterophorum are well established in the lower Himalaya due to suitable environmental conditions for their growth as similar to their native range [18] [20]. Due to the higher level of invasion which was found gradually decreased the human interference from industrial areas to highly populated inhabitancy areas, high traffic highway road sides, industrial link roads, rangelands, semi-urban areas, minor highways, link roads between urban areas and village, agricultural lands of urban areas, these areas were found under the threat of high invasion unless proper management was undertaken.

\section{References}

[1] GEF (2003) Removing Barriers to Invasive Plants Management in Africa. Under the PDF-V Phase of UNEP and GEF Project (Global Environmental Faciliities), Nairobi Kenya.

[2] Tefera, T. (2002) Allelopathic Effects of Parthenium hysterophorus Extracts on Seed Germination and Seedlings Growth of Eragrostis tef. Journal of Agronomy and Crop Science, 188, 306-310. http://dx.doi.org/10.1046/j.1439-037X.2002.00564.x

[3] Bhowmik, P.C. and Sarkar, D. (2005) Parthenium hysterophorus L.: Its World Status and Potential Management. Proceeding of the Second International Conference on Parthenium Management, Bangalore, 5-7 December 2005, 1-6.

[4] Mack, R.N. and Lonsdale, W.M. (2001) Humans Are Global Plant Dispersers: Getting More than We Have Bargained for. Bioscience, 51, 95-102. http://dx.doi.org/10.1641/0006-3568(2001)051[0095:HAGPDG]2.0.CO;2

[5] Dhileepan, K. (2009) Managing Parthenium Weed across Diverse Landscapes: Prospects and Limitations. Management of Invasive weeds, 5, 227-259.

[6] Dhawan, S.R. and Dhawan, P. (1996) Regeneration in Parthenium hysterophorus L. World Weeds, 3, 181-182.

[7] Anonymous (2005) Invasive Alien Species. Convention on Biological Diversity. CBD (Convention on Biological Diversity).

[8] Curtis J.T. (1959) The Vegetation of Wisconsin: An Ordination of Plant Communities. University of Wisconsin Press, Madison, 657.

[9] Curtis, J.T. and McIntosh, R.P. (1950) The Interrelations of Certain Analytic and Synthetic Phyto-Sociological Characters. Ecology, 31, 434-455. http://dx.doi.org/10.2307/1931497

[10] Phillips, E.A. (1959) Methods of Vegetation Study. Holt, Rinehart and Winston, New York, 107 p.

[11] Kohli, R.K., Batish, D.R., Singh H.P. and Dogra. K.S. (2006) Status, Invasiveness and Environmental Threats of Three Tropical American Invasive Weeds (Parthenium hysterophorus L., Ageratum conyzoides L., Lantana camara L.) in India. Biological Invasions, 8, 1501-1510. http://dx.doi.org/10.1007/s10530-005-5842-1

[12] Adkins, S.W. and Sowerby M.S. (1996) Allelopathic potential of the weed Parthenium hysterophorus L. in Australia. Plant Protection Quarterly, 11, 20-23. 
[13] Van Wilgen, B.W. and Van Wyk, E. (1999) Invading Alien Plant in South Africa: Impacts and Solutions. VIth International Rangeland Congress Proceedings, 2, 566-571.

[14] Timsina, B. (2007) Impact of Parthenium hysterophorus L. Invasion on Soil and Plant Species Composition of Grasslands of Central Nepal. M.Sc. Dissertation, Tribhuvan University, Kathmandu, 61 p.

[15] Mahadevappa, M., Das, T.K. and Kumar, A. (2001) Parthenium: A Curse for Natural Herbs. National Research Seminar on Herbal Conservation, Cultivation, Marketing and Utilization with Special Emphasis on Chhattisgarh, Raipur, 13-14 December 2001, 9-10.

[16] Wahab, S. (2005) Management of Parthenium through an Integrated Approach Initiatives, Achievements and Research Opportunities in India. Proceeding of the Second International Conference on Parthenium Management, Bangalore, 57 December 2005, 55-59.

[17] Mulisa, U., Taye, T. and Firehun, Y. (2008) Impact of Parthenium hysterophorus L. on Herbaceous Plant Diversity in Range Lands of Fentale District in the Central Rift Valley of Ethiopia. Ethiopia Journal Weed Management, 2, 13-29.

[18] Dogra, K.S. Kohli, R.K. and Sood, S.K. (2009a) An Assessment and Impact of Three Invasive Species in the Shivalik Hills of Himachal Pradesh, India. International Journal of Biodiversity Conservation, 1, 4-10.

[19] Kohli, R.K. Batish, D.R., Singh, H.P. and Dogra, K.S. (2009) Ecological Status of Some Invasive Plants of Shiwalik Himalayas in Northwestern India. In: Kohli, R., Jose, S., Batish, D. and Singh, H.P., Eds., Invasive Plants and Forest Ecosystem, CRC/Taylor Press, Netherlands, 143-156.

[20] Dogra, K.S. Kohli, R.K., Sood, S.K. and Dobhal, P.K. (2009) Impact of Ageratum conyzoides L. the Diversity and Composition of Vegetation in the Shivalik Hills of Himachal (Northwestern Himalaya). International Journal of Biodiversity Conservation, 1, 135-145.

\section{Abbreviations}

IVI = Importance Value Index

$\mathrm{RD}=$ Relative Density

$\mathrm{RF}=$ Relative Frequency

KT = Kota site, KT1-5 SK1-5 for five sites each invaded and non invaded sites

SK = Sakri, SK1-5 for five sites each invaded and non invaded sites

$\mathrm{KN}=$ Koni, KN1-5 SK1-5 for five sites each invaded and non invaded sites 
Scientific Research Publishing (SCIRP) is one of the largest Open Access journal publishers. It is currently publishing more than 200 open access, online, peer-reviewed journals covering a wide range of academic disciplines. SCIRP serves the worldwide academic communities and contributes to the progress and application of science with its publication.

Other selected journals from SCIRP are listed as below. Submit your manuscript to us via either submit@scirp.org or Online Submission Portal.
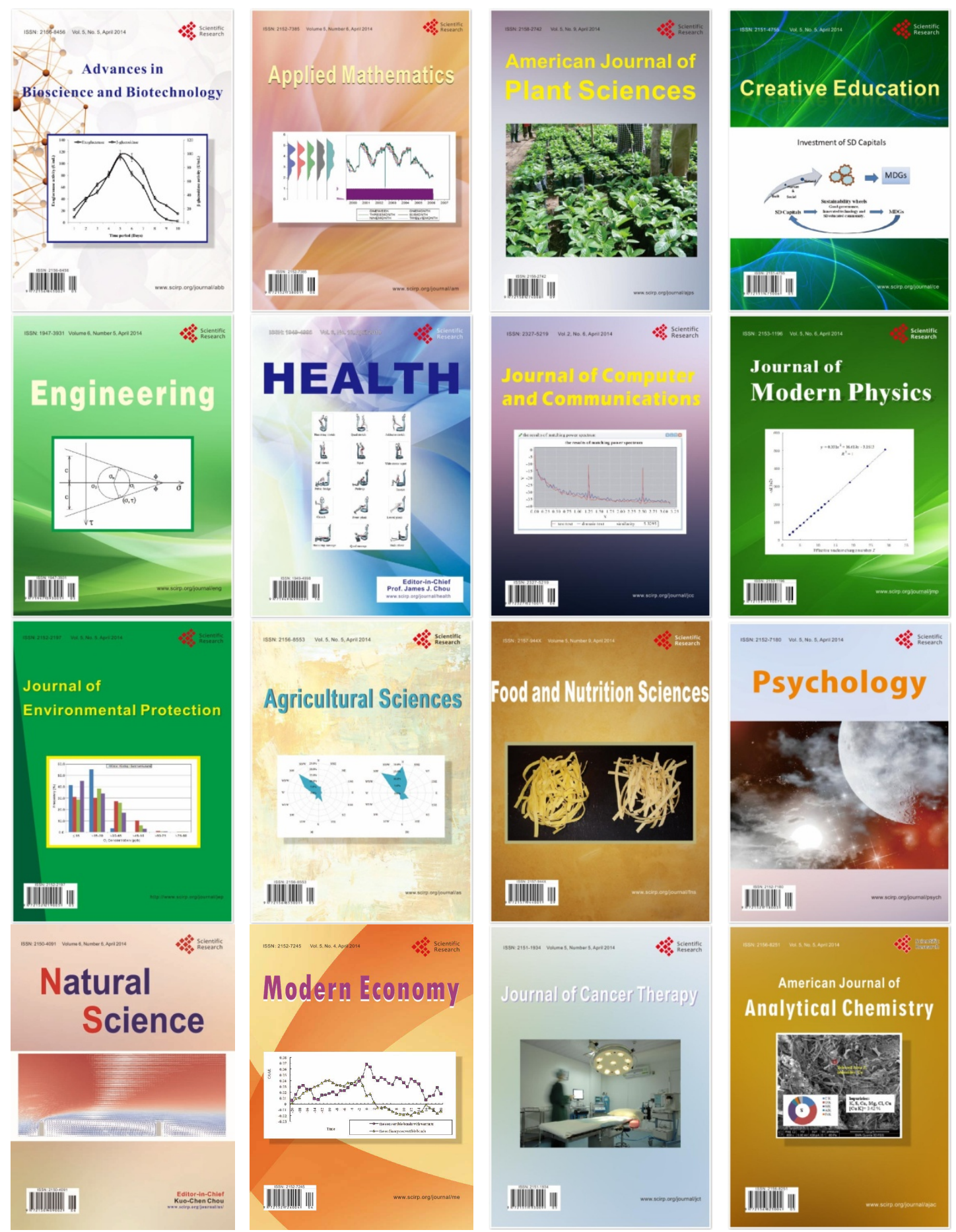